\title{
Pioneer Growth Cone Adhesion in vivo to Boundary Cells and Neurons After Enzymatic Removal of Basal Lamina in Grasshopper Embryos
}

\author{
Maureen L. Condic ${ }^{1}$ and David Bentley ${ }^{2}$ \\ 'Neurobiology Group and 'Department of Molecular and Cell Biology, University of California, Berkeley, California 94720
}

The Ti1 pioneer neurons of embryonic grasshopper limbs extend axons between the limb epithelium and its basal lamina. Their growth cones exhibit high affinity for both limb segment boundaries and immature neurons. We have investigated the role of the basal lamina in growth cone adhesion to neurons and segment boundaries by removing the basal lamina with mild enzymatic digestion when the Tit growth cones are in contact with different cellular substrates.

If the basal lamina is removed when the Ti1 growth cones are in contact with other neurons, the growth cones remain in contact with the neuronal somata, and the Ti1 cell bodies reposition proximally. This suggests that the basal lamina is involved in the adhesion of the Ti1 somata to the substrate but not in growth cone-neuronal adhesion. This is the first direct evidence that growth cones establish adhesive cellcell interactions with other neurons in vivo.

Enzymatic treatments that remove the basal lamina also cause embryonic limbs to elongate. If the $\mathrm{Ti} 1$ axons are strongly apposed to 2 segment boundaries prior to protease treatment, their somata reposition to the nearest segment boundary, yet their axons do not retract off of the segment boundaries, despite severe stretching by the enzyme-induced limb expansion. These results indicate that the affinity of the Ti1 cells for segment boundaries is due at least in part to adhesive cell-cell interactions that are resistant to proteolytic digestion and independent of the basal lamina.

Basal laminae and extracellular matrix proteins have been postulated to play crucial roles in axon guidance in a number of different systems (Hay, 1981; Sanes and Chiu, 1983; Sanes and Covault, 1985; Bixby et al., 1987; Jessell, 1988). It is well established that neurons express specific cell and substrate recognition molecules that may mediate axon guidance during development (see Obrink, 1986; Hyncs, 1987; Jessell, 1988, for reviews). However, little is known about the contribution of the basal lamina to axon guidance in vivo, as growth cones encounter different combinations of cell surface and extracellular matrix

\footnotetext{
Received Scpt. 30, 1988; revised Dec. 20, 1988; accepted Jan. 20, 1989.

We thank Dr. D. Fristrom and Dr. H. J. Yost for their critical reading of the manuscript, and H. J. Yost for assistance with the summary diagram. The scanning electron microscopy was done with the excellent technical assistance of A. ToroianRaymond. This work was supported by an NSF predoctoral grant and an NIH predoctoral training grant (T32-GM07048-14) to M.L.C.: NIH Jacob Javits grant (NS09094-19) and March of Dimes Birth Defects Foundation grant $(1-1089)$ to D.B.

Correspondence should be addressed to Maureen L. Condic at the above address. Copyright ic 1989 Society for Ncuroscience $0270-6474 / 89 / 082687-10 \$ 02.00 / 0$
}

components. Pioneer neuron pathfinding affords an opportunity to investigate the contribution of the basal lamina to axon guidance in a simple in vivo system, where several axonal guidance cues have been well described.

The embryonic grasshopper limb bud consists of a simple epithelial cone surrounding a monolayer of undifferentiated mesodermal cells with a basal lamina separating the mesodermal and epithelial cell layers (Wigglesworth, 1953; Ashhurst, 1965, 1982). The basal lamina appears to be deposited by both epithelial cells and mesodermal hemocytes (Ball et al., 1987; Mirre et al., 1988). A number of molecular components common to vertebrate laminac occur in insects, including collagen type IV (Blumberg et al., 1987). laminin (Fessler et al., 1987; Montell and Goodman, 1988), fibronectin (Grateios et al., 1988), and a nidogen-entactin-like compound (Blumberg et al., 1987). In addition, molecules homologous to several vertebrate substrate receptors have been described in insects (Bogaent et al., 1987; I eptin et al., 1987; MacKrell ct al., 1988).

At $30 \%$ of development the Til cells arise at the tip of the limb, emerge from the epithelium and extend axons between the basal lamina and the epithelial endfeet (Bate, 1976; Keshishian, 1980; Lefcort and Bentley, 1989). The growth cones extend numerous filopodia betwcen the epithelial cclls and through the basal lamina into the mesodermal cell layer (Caudy and Bentley, 1986a). The Til axons pioneer the first neuronal pathway observed in limb buds (Bate, 1976; Keshishian, 1980). The route taken by the growth cones of the Til neurons is highly stereotyped, and forms the basis of one of the major nerve trunks in the adult animal (Bentley and Keshishian, 1982; Ho and Goodman, 1982; Keshishian and Bentley, 1983).

Some features of the limb that orient growth cones have already been identified. At specific axial locations, the Til growth cones selectively recognize and reorient towards the cell bodies of other immature neurons in the limb (Ho and Goodman, 1982; Bentley and Keshishian, 1982; Keshishian and Bentley, 1983; Bentley and Caudy, 1983a, b; Caudy and Bentley, 1986b). One pair of nascent neurons, the $\mathrm{Cx} 1$ cells, appears to be necessary for the Til growth cones to establish their normal connection to the CNS (Bentley and Caudy, 1983a). The Til growth cones also selectively recognize and reorient along segment boundaries (Bentley and Caudy, 1983b; Caudy and Bentley, 1987). When the $\mathrm{Til}$ growth cones are not in contact with either neurons or limb segment boundaries, a proximally increasing gradient of affinity may orient the growth cones (Caudy and Bentley, 1986a). Neural-substrate interactions are also important for axon guidance in Drosophila wing disks (Blair and Palka, 1985; Blair ct al., 1985, 1987; Palka, 1987). The information necessary for 
Table 1. Enzyme effects on basal lamina and on neuronal disposition

\begin{tabular}{lllll} 
Enzyme & $\begin{array}{l}\text { Embryo } \\
\text { stage } \\
(\%)^{a}\end{array}$ & $\begin{array}{l}\text { Enzyme } \\
\text { conc. } \\
(\%)\end{array}$ & $\begin{array}{l}\text { Disruption of } \\
\text { basal lamina } \\
(n)^{b}\end{array}$ & $\begin{array}{l}\text { Cell } \\
\text { body } \\
\text { relocation }\end{array}$ \\
\hline Control & 34.5 & $-{ }^{d}$ & $0(4)$ & $0 / 12$ \\
& 36.5 & - & $0(7)$ & - \\
Trypsin & 34.5 & $0.02-0.04$ & $0 /+(6)$ & $1 / 1$ \\
Elastase & 34.5 & $0.1-0.25$ & $+++(6)$ & $9 / 12$ \\
& 36.5 & $0.1-0.25$ & $+++(10)$ & - \\
Ficin & 36.5 & $0.02-0.04$ & $+++(8)$ & -
\end{tabular}

${ }^{a}$ Percentage of complete development $( \pm 0.5)$.

"Evaluated in SEM: 0, no effect, + , slight removal, ++ , less than complete removal, +++ , complete removal.

"Number of embryos with $\geq 50 \%$ axon pairs affected per total number of embryos examined.

"Not applicable or not measured.

the proximal growth of the Til axons, recognition of the limb segment boundaries and the $\mathrm{Cx} 1$ guidepost neurons is contained within the limb epithelium, its basal lamina, and the neurons themselves (Lefcort and Bentley, 1987). For none of these guidance cues is it known whether the orienting information is provided by cell-surface or extracellular matrix molecules.

We have recently demonstrated that mild enzymatic digestion with elastase, ficin, or papain completely removes the basal lamina from embryonic grasshopper limbs as viewed in scanning electron microscopy. Growth cone-basal lamina interactions were investigated by removing the basal lamina from limb buds and observing the immediate response of the Til growth cones. These experiments suggest that the Til axons are under tension in vivo and that growth cone-basal lamina adhesive interactions support initial axon outgrowth in this system (Condic and Bentley, 1989).

In the work reported here we have examined the effect of removing the basal lamina at different stages of development on the immediate response of the Til neurons in vivo, when their growth cones are in contact with guidepost neurons and the limb segment boundaries.

\section{Materials and Methods}

Embryos of Schistocerca americana were obtained from a colony maintained at the University of California, Berkeley. They were staged by percentage of total embryonic development completed (Bentley et al., 1979; Caudy and Bentley, 1986a). Limb axes, specific neurons, and segment boundaries are named as in Caudy and Bentley (1986a; for depiction of embryonic vs adult limb axes, see Ball and Goodman, 1985). The principal neurons are labeled in Figure $1 A$. The limb segment boundaries are labeled in Figure $4 B$.

Enzyme treatments. Trypsin (T-8253), elastase (E-0258), and ficin (F-6008) were obtained from Sigma. The enzymes were stored as $0.5 \%$ solutions in saline $\left(\mathrm{NaCl} 150 \mathrm{~mm}, \mathrm{CaCl}_{2} 4 \mathrm{~mm}, \mathrm{KCl} 10 \mathrm{~mm}, \mathrm{MgCl}_{2} 2\right.$ $\mathrm{mm}$, TES $5 \mathrm{~mm}$, sucrose $140 \mathrm{~mm}, 0.1 \% \mathrm{BS} \Lambda$, pH 7.2) at $-20^{\circ} \mathrm{C}$. For each enzyme and for each embryonic stage considered, comparable effective enzyme concentration ranges were determined as described previously (Condic and Bentley, 1989). The enzyme concentrations used in these experiments are reported in Table 1. Embryos were dissected in saline, opened dorsally to allow enzyme access to the limb lumen and incubated for $2.5-3 \mathrm{hr}$ at $30^{\circ} \mathrm{C}$ (Table 1 ).

Scanning electron microscopy. To observe neuron-neuron and neuron-segment boundary interactions after removal of the basal lamina, the mesoderm was removed from the right metathoracic leg with a suction pipette (Lefcort and Bentley, 1987), allowed to recover for $1 \mathrm{hr}$ in saline at $30^{\circ} \mathrm{C}$, then exposed to enzyme or saline solution for $1.5-2.5$ $\mathrm{hr}$ and processed immediately for SEM as described previously (Condic and Bentley, 1989). Note: The shorter incubation period used for SEM preparations reflects the shorter amount of time required for the enzyme solutions to diffuse into limbs from which the mesoderm had been removed. The preparations were viewed in an ISI-DS-130 SEM. Legs from 41 embryos were examined (lable 1 ).

Immunofluorescent labeling of neurons in whole-mounted embryos. The effects of enzyme incubation on neuronal disposition were determined in immunofluorescence labeled embryos. Embryos were exposed to enzyme as described above (see Table 1). Immediately following incubation, the embryos were fixed overnight in $4 \%$ formaldehyde in saline (without sucrose) and labeled with anti-HRP antibodies according to the protocol of Caudy and Bentley (1986a). These antibodies selectively label insect neurons (Jan and Jan, 1982; Snow et al., 1987). Embryos were viewed and photographed in whole-mount with a Zeiss epifluorescence microscope. Over 150 embryos were examined, and data from 39 are presented in Table 1.

Quantification of Til somata relocation at the $34.5 \%$ stage. To quantify the change in position of the Til cell bodies, embryos from a single clutch of eggs were staged to $34.5 \pm 0.5 \%$ of development and incubated in either elastase $(0.1 \%)$, trypsin $(0.02 \%)$, or saline. The embryos were fixed, labeled with anti-HRP antibody, and viewed in whole-mount. Measurements were made using an ocular micrometer. The length of the axon was determined by measuring from the midpoint of the Til cell body pair to the farthest proximal process of their growth cones that was not a filopodium. The distance between this point on the growth cones and the center of the Cxl cell body pair was also measured. The distances between all the neuronal somata in the limb were measured from the center of each cell soma or the midpoint of the paired $\mathrm{Cxl}$ and Til cells. Measurements were taken only from those legs in which the Til, Fe1, Tr1, and $\mathrm{Cx} 1$ neurons were clearly visible with the antiHRP antibody.

The enzymatic digestions used in these experiments resulted in enzyme specific changes in the size and shape of limbs at this stage. Elastolytic digestion caused a pronounced elongation and thinning of the limb, whereas trypsin caused the legs to constrict at the segment boundaries. Consequently, the absolute distance between homologous points in the limb was not constant across experimental conditions. The relative positions of the $\mathrm{Fe} 1, \operatorname{Tr} 1$, and $\mathrm{Cx} 1$ neurons were not affected, however, since the ratio of the distance between (Fel-Trl) and the distance between $(\operatorname{Tr} 1-\mathrm{Cx} 1)$ did not differ significantly between experimental conditions ( $p>0.4, t$ test; see Fig. 2 for sample sizes). These measurements were therefore used to scale the absolute measurements.

Quantification of limb elongation at the $36 \%$ stage. To quantify the extent of limb elongation observed with elastase treatment, embryos from 3 clutches of eggs at $36-37 \%$ of development were treated with elastase $(0.1 \%)$ for $2.5 \mathrm{hr}$ as described above, fixed, and labeled with the anti-HRP antibody. The distance from the center of the Trl neuron to the center of the Til neuronal pair (Tr1-Ti1) and the distance from the $\operatorname{Tr} 1$ neuron to the tip of the limb (Trl-limb tip) were measured in 8 control and 8 experimental embryos (48 axon pairs each). The Tr $1-$ Til distance showed an average increase of $15 \%$ in the elastase-treated animals relative to the average control value. The Tr1-limb tip distance showed an average increase of $17 \%$ in elastase treated versus control embryos, with some protease-treated individuals more than $5 \mathrm{SD}$ above the control mean. The variability of both measurements was also increased by elastase treatment. These data are summarized in Table 2 .

\section{Results}

\section{Effects of enzymatic digestion on limb epithelium}

It has previously been established that the enzymatic treatments used in these experiments remove the basal lamina from embryonic limbs without seriously affecting the viability of either the limb epithelium or the neurons (Condic and Bentley, 1988, 1989). Protease digestion at the $34.5 \%$ stage or older resulted in enzyme-specific changes in limb shape that were evident after a $1 \mathrm{hr}$ exposure to the standard enzyme concentrations. Elastase consistently caused an increase in the length and concomitant decrease in the diameter of the limb (Table 2). This effect was also seen with ficin. In order to show all limbs at the same magnification, only embryos with mild elongation were selected for illustrations. However, elastase- and ficin-associated limb elongation could be considerable, with increases of $30-50 \%$ in 
Table 2. Elastase-induced limb elongation at the $36 \%$ stage

\begin{tabular}{|c|c|c|c|c|}
\hline \multirow[b]{2}{*}{ Condition } & \multicolumn{2}{|c|}{ Tr1 to Til distance ${ }^{\alpha}$} & \multicolumn{2}{|c|}{$\operatorname{Tr} 1$ to limb tip distance ${ }^{a}$} \\
\hline & $\begin{array}{l}\text { Average } \\
\text { distance }\end{array}$ & $\begin{array}{l}\text { Range } \\
(\min -\max )\end{array}$ & $\begin{array}{l}\text { Average } \\
\text { distance }\end{array}$ & $\begin{array}{l}\text { Range } \\
(\min -\max )\end{array}$ \\
\hline & $180 \pm 37$ & $123-269$ & $400 \pm 57$ & $291-537$ \\
\hline Elastase $^{b}$ & $203 \pm 46^{c}$ & $123-323$ & $469 \pm 74$ & $346-700$ \\
\hline
\end{tabular}

Eight animals from 3 different clutches of eggs were measured for both control and elastase conditions (48 limbs in each condition).

${ }^{\alpha}$ Distance is measured in $\mu \mathrm{m} \pm \mathrm{SD}$.

blastase at $0.1 \%$ for $2.5 \mathrm{hr}$.

'Significantly different from control values at $p<0.0001$, ANOVA.

length not uncommon (see Table 2). Trypsin had more subtle effects on overall limb morphology, resulting in a slight constriction of the limb at the segment boundaries. For all enzymes, the effects on limb morphology varied in magnitude between individuals. This variation may reflect real differences between individuals in the developmental state of the epithelium and/ or basal lamina relative to the general time course of neuronal development (see Caudy and Bentley, 1986a). At the standard enzyme concentrations used for these experiments, all enzymeinduced changes in limb shape occurred without obviously affecting the integrity of the epithelium.

\section{Changes in the position of the Til cell bodies}

By the $34 \%$ stage of development, the Til neurons have grown proximally, contacted the Fel and $\mathrm{Tr} 1$ guidepost neurons, reoriented at the coxa/trochanter $(\mathrm{Cx} / \mathrm{Tr})$ segment boundary, crossed over into the ventral coxa, and contacted the $\mathrm{Cx} 1$ neurons (Keshishian and Bentley, 1983; Caudy and Bentley, 1986b; Fig. $1 A$ ). When limbs at this stage of development were treated with concentrations of elastase or ficin known to remove the basal lamina, the Til somata were observed in more proximal positions; adjacent to the Fel neuron, between the Fel and $\operatorname{Tr} 1$ neurons and in some cases adjacent to the $\operatorname{Tr} 1$ neurons (Figs. $1 C, 4 F)$. Although treatment with trypsin caused the limbs to constrict at the segment boundaries and contract somewhat in length, Ti1 neurons did not reposition after trypic digestion (Fig. 1B).

\section{Quantification of Til cell body relocation}

To quantify the change in position of the Til somata, a clutch of eggs was selected in which the Til axons had grown past the Cx1 neurons towards the CNS, but neither Fel nor Tr1 had begun axonogenesis (34.5\% of development). Embryos were exposed to either elastase, trypsin, or saline, then fixed and labeled with anti-HRP antibodies. The length of the axons, the distance between the proximal tip of the Til growth cones and the Cx1 neurons, and the distances between the cell bodies of all of the other neurons in the limb at this stage ( $\mathrm{Fe} 1$ and $\operatorname{Tr} 1, \operatorname{Tr} 1$ and $\mathrm{Cx} 1)$ were measured. These results are summarized in Figure 2. For all conditions the absolute distances have been scaled to account for systematic changes in limb size due to the enzymatic treatment (see Fig. 2; Materials and Methods). Removal of the basal lamina by elastase treatment resulted in a pronounced decrease in the length of the Til axons relative to the length of the limb. Most of the decrease in axon length was due to proximal movement of the Til cell bodies. Trypsin treatment did not significantly alter the length of the axons relative to the size
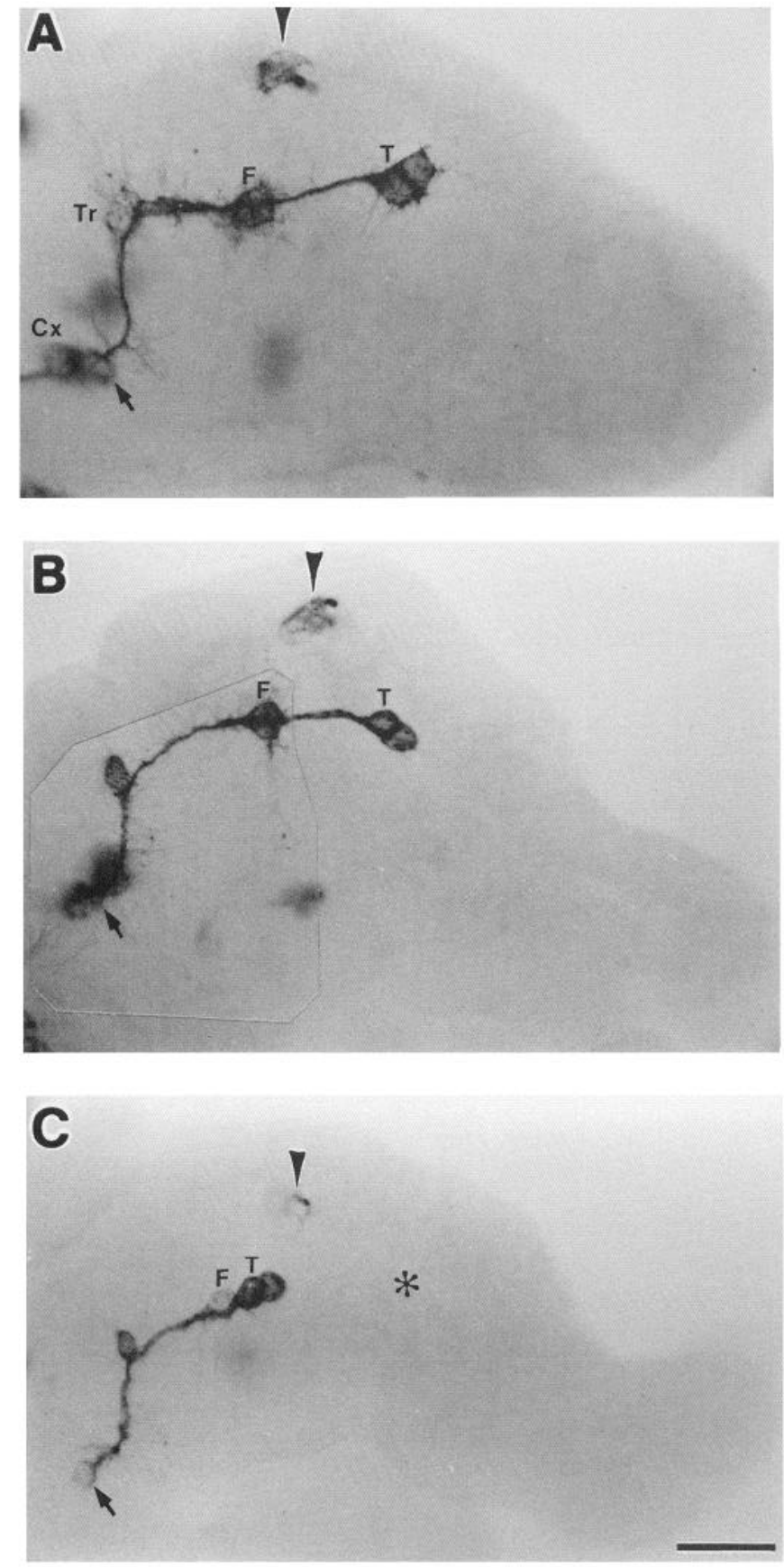

Figure 1. Ti1 neurons in $34.5 \%$ stage metathoracic limbs after enzymatic treatment. The neurons are stained with anti-HRP antibodies, and viewed in whole-mount ( $T=$ Til pioneers; $F=\mathrm{Fe} 1$ neuron; $T r=$ $\operatorname{Tr} 1$ neuron; $C x=\mathrm{Cx} 1$ neurons; arrowhead $=$ femoral chordotonal organ neurons; small arrow $=$ Til growth cones contacting the $\mathrm{Cx} 1$ neurons). $A$, Til pathway in a control limb. $B$, Til pathway after treatment with $0.01 \%$ trypsin for $2.5 \mathrm{hr}$. The relative position of the Til cells to the other neurons of the limb does not change. $C$, Til pathway after treatment with $0.1 \%$ elastase for $2.5 \mathrm{hr}$. The growth cones remain in contact with the $\mathrm{Cxl}$ neurons, but the Til somata have relocated proximally, to lie adjacent to $\mathrm{Fel}$. The asterisk indicates the approximate initial position of the Til somata. Dorsal is $u p$; distal is to the right. Limbs are from the same clutch of eggs used for the quantification presented in Figure 2. Scale bar, $50 \mu \mathrm{m}$. 


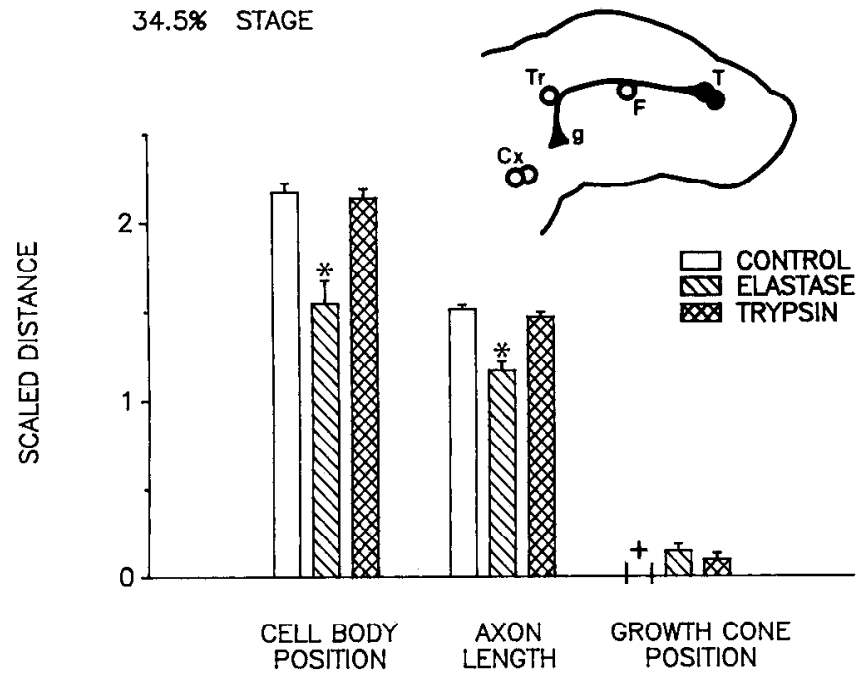

Figure 2. Quantification of neuron disposition after incubation with elastase and trypsin. Measurements were made on anti-HRP antibody labeled, whole-mounted embryos taken from a single clutch of eggs. The group means (bar) and SEM (T) are presented. Embryos at the $34.5 \%$ stage were exposed for $2.5 \mathrm{hr}$ to either saline (control, $n=33$ ), $0.1 \%$ elastase $(n=37)$, or $0.02 \%$ trypsin $(n=36)$. The distances reported are scaled to compensate for differences in limb shape (see Materials and Methods). Cell body position is the distance between the Trl soma $(T r)$ and the Ti1 soma ( $T$; see inset), scaled by the $\operatorname{Tr}$ to Fel soma $(F)$ distance: (TR to $\mathrm{T}) /(\mathrm{Tr}$ to $\mathrm{F})$. Axon length is the distance from the Ti1 somata and their growth cones $(g)$, scaled by the distance from $F$ to $\mathrm{Tr}$ to $\mathrm{Cx}:(\mathrm{T}$ to $\mathrm{g}) /(\mathrm{F}$ to $\mathrm{Tr}+\mathrm{Tr}$ to $\mathrm{Cx})$. Growth cone position is the distance between the growth cones $(g)$ and $\mathrm{Cx}$ cells scaled by $\mathrm{F}$ to $\mathrm{Tr}$ to $\mathrm{Cx}$ distance: $(\mathrm{g}$ to $\mathrm{Cx}) /(\mathrm{F}$ to $\mathrm{Tr}+\operatorname{Tr}$ to $\mathrm{Cx})$. The asterisk indicates a significant difference from controls at $p<0.0001$, ANOVA; + , indicates the control value is equal to zero.

of the limb, nor did it change the relative position of either the Til growth cones or cell bodies.

\section{Interactions of Ti1 cells with neuronal cell bodies in SEM}

In the majority of limbs observed, the Ti1 growth cones remained in contact with the $\mathrm{Cx} 1$ neurons after removal of the basal lamina. To observe these neural-neural interactions at higher resolution, we examined the Til and other neuronal cell bodies in embryos from the $32-34.5 \%$ stage using scanning electron microscopy (Fig. 3). The neurons were identified in these preparations by several criteria: their adepithelial location, their cell number (single cells for $\mathrm{Tr} 1$ and Fel, paired for the Til and $\mathrm{Cx} 1$ cells), their characteristic position along the limb axis (tibia, femur, trochanter, and coxa), their characterisitc circumferential location (all neurons are located on the anterior limb face; Til, $\mathrm{Fe} 1$, and $\mathrm{Tr} \mathrm{l}$ are in the dorsal quadrant, $\mathrm{Cx} 1$ in the ventral quadrant), and the orientation of their somata relative to the limb axis ( $\mathrm{T} i 1, \mathrm{Fe} 1$, and $\mathrm{Cx} 1$ cells oriented longitudinally, $\operatorname{Tr} 1$ oriented circumferentially). The Til cells could also be identified by virtue of their being the only cells in the limb with axons at this stage of development.
After elastase digestion the Til axons often had few filopodia or branches remaining, suggesting they remained quite weakly attached to the substratum (see Figs. $1 C, 4 B$ ). Consistent with these observations, preserving the axons of the Til neurons in their final configuration after removal of the mesoderm and basal lamina proved to be quite difficult. We were able however, to observe several cases of the Til cells interacting directly with other identified neurons in the limb after complete removal of the basal lamina using standard enzyme concentrations (Fig. 3, $A, F)$.

We also attempted to preserve the Til axons by conducting a more limited digestion (ficin $0.04 \%, 1-1.5 \mathrm{hr}$ ) on limbs at the $36-37 \%$ stage. With this approach, we observed several cases in which the basal lamina was largely removed from a portion of the Til pathway, such that neural-neural interactions could be directly observed (Fig. 3, $B-E$ ).

The axons, growth cones, fine branches, and filopodia of the Til neurons remained tightly apposed to other neurons after removal of the basal lamina. Several types of neuronal cell-cell appositions were observed. The somata of the Ti1 and $\mathrm{Cx} 1$ cell pairs remained tightly apposed to each other (Fig. 3, $A, C, F$; occasionally, separated Til somata were observed, at frequencies comparable to those seen in control embryos, see Fig. $3 E$ ). Axon-axon fasciculation was maintained (Fig. 3, B, D, F). Axonsoma interactions were observed between the Til axons and the Fel neuron (Fig. $3 B$ ). The Til growth cones remained in contact with the Cx1 somata (Fig. $3 F$ ). In many cases, filopodia from the Til cells remained apposed to the somata of other neurons (Fig. 3, $B-D$ ).

\section{Interactions of Til axons and growth cones with segment boundaries}

In normal limbs, the Ti1 growth cones often cross the femur/ tibia ( $\mathrm{Fe} / \mathrm{Ti}$ ) segment boundary at the $31 \%$ stage without responding to it in any overt manner. By the $36 \%$ stage, when the Til axons have entered the CNS, virtually all of the axons observed crossing this boundary show pronounced spreading and filopodial retention in this region of the limb (Caudy and Bentley, 1986a, 1987; Fig. 4A). The Til response to the trochanter/femur $(\mathrm{Tr} / \mathrm{Fe}$ ) segment boundary at the $36-37 \%$ stage is very similar to their response at the Fe/ Ti boundary; filopodia and branches are preferentially extended and/or retained in this region, usually in a dorsal direction. The axons often show a kink at this boundary (Fig. $4 A$ ). At this stage, the Til cell bodies and some of the distal portions of their axons begin to emerge through the basal lamina into the limb interior (Fig. 4C).

When the basal lamina was removed from embryonic limbs at the $36 \%$ stage by treatment with elastase or ficin, the interactions of the Til axons with segment boundaries persisted. Axonal lamellae and filopodia were retained on the $\mathrm{Fe} / \mathrm{Ti}$ boundary, although the number and length of the filopodia observed were reduced and the tips of the filopodia sometimes appeared beaded, suggesting partial retraction (Fig. 4B). The interactions of the Til axons with the $\mathrm{Tr} / \mathrm{Fe}$ boundary also

Figure 3. Scanning electron micrographs of identified neurons in enzyme treated limbs. The mesoderm was removed before treatment, and the limbs were fixed immediately after treatment. $A$, Til neurons in a $32 \%$ stage limb after treatment with $0.02 \%$ ficin for $2 \mathrm{hr}$. The basal lamina has been completely removed. The somata are tightly apposed to each other and lie on a field of epithelial basal endfeet $(e)$ over which they extend some processes (arrowheads). The growth cones are withdrawn almost to the somata (arrow). $B$, The 2 Til axons (large arrows) extending over the Fel neuron after exposure to $0.04 \%$ ficin for $70 \mathrm{~min}$ in a limb at the $36-37 \%$ stage of development. The basal lamina overlying Fel has been 

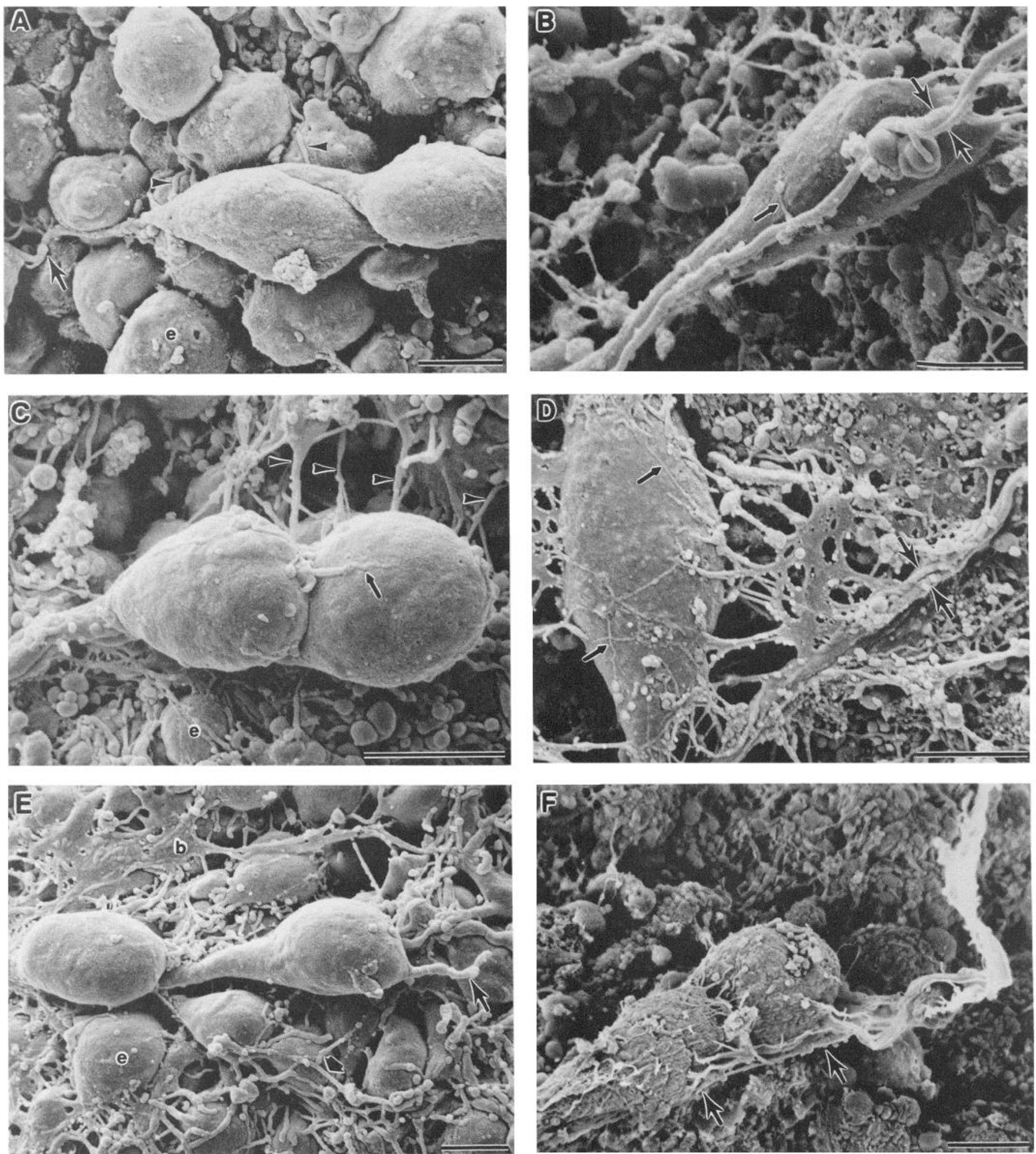

completely removed. Processes (e.g., small arrow) extending from the axons remain tightly apposed to the Fel soma. $C$, The Til somata after treatment with $0.04 \%$ ficin for $70 \mathrm{~min}$ in a $36-37 \%$ stage limb. The basal lamina has been completely removed. A neuronal process (small arrow) from the proximal pioneer remains extended over the distal pioneer. Neuronal processes (arrowheads) remain in contact with epithelial endfeet (e.g., $e$ ) in the region of the $\mathrm{Fe} / \mathrm{Ti}$ boundary. $D$, The $2 \mathrm{Til}$ axons (large arrows) interacting with the Trl neuron after treatment with $0.04 \%$ ficin for $70 \mathrm{~min}$ in a $36-37 \%$ stage limb. The basal lamina has been only partially removed by this treatment but is completely removed from much of the $\operatorname{Tr} 1$ soma. Til filopodia normally wrap $\operatorname{Tr} 1$ (see Figs. $1 A, 4 A$ ); fine processes (small arrows), possibly originating from the Til axons, remain extended over the $\operatorname{Tr} 1$ soma in regions devoid of basal lamina. E, Separated Til somata after treatment with $0.04 \%$ ficin for 50 min in a $36-37 \%$ stage limb. The basal lamina has been only partially removed by this treatment $(b)$. The distal pioneer retains a short distal process (arrow) that extends across epithelial endfeet (e). Numerous epithelial endfeet processes are visible (e.g., broad arrowhead). F, Til growth cones (arrow) interacting with the $\mathrm{Cx} 1$ neurons after treatment with $0.075 \%$ elastase for $1 \mathrm{hr}$ in a $34.5 \%$ limb. The growth cones are tightly apposed to the paired Cx1 somata (arrows), although the basal lamina has been removed. Scale bars, $5 \mu \mathrm{m}$. 

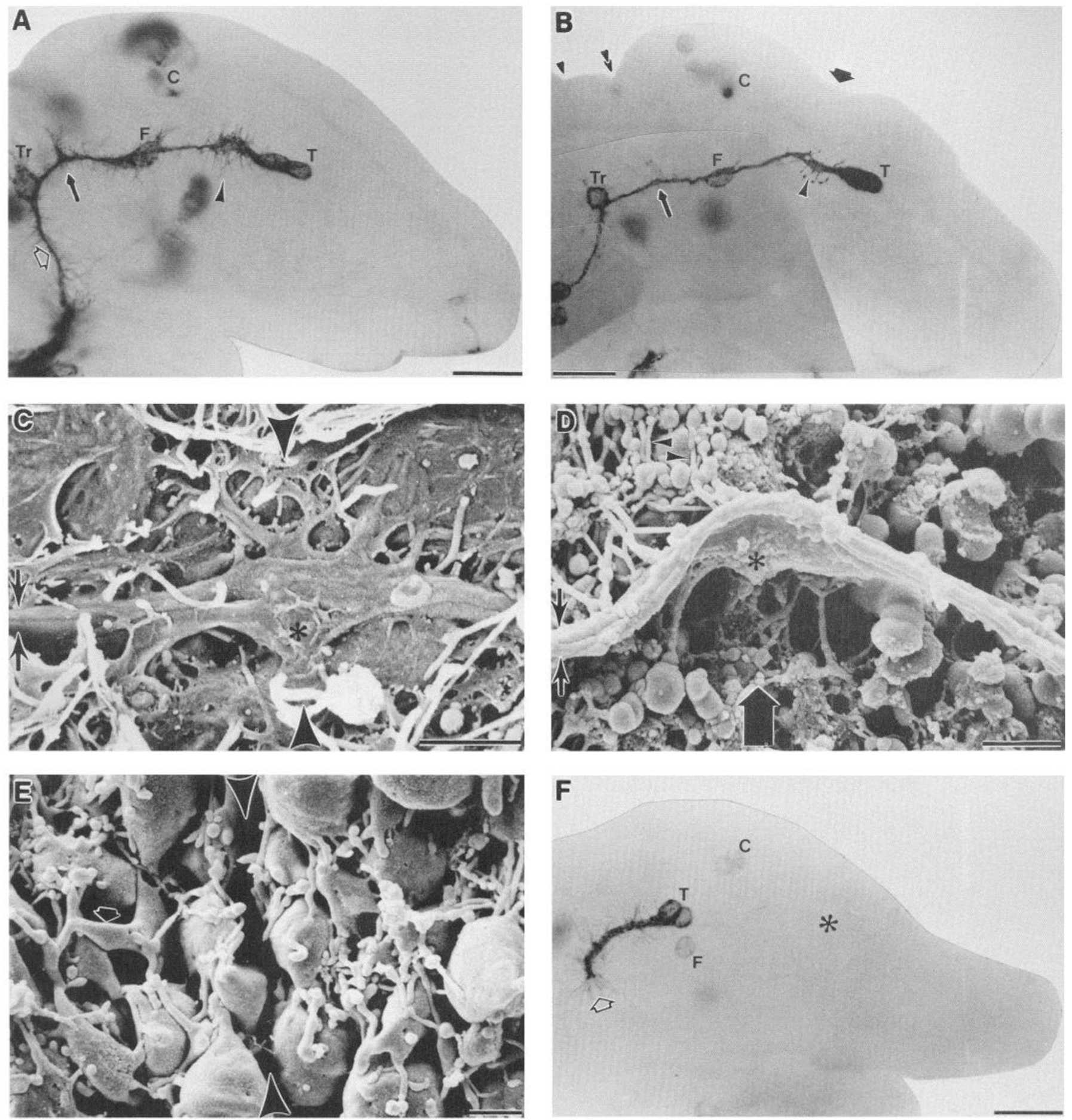

Figure 4. Interactions of the Til neurons with limb segment boundaries after enzymatic treatment. $A, B$, and $F$, Whole-mounts of limbs stained with the anti-HRP antibody $(C$, femoral chordotonal organ; $T$, Til neurons; $F$, Fel neuron; $T r$, Tr1 neuron). $C, D$, and $E$, Scanning electron micrographs of segment boundary regions. $A$, Til pathway in a control limb at the $36-37 \%$ stage of development. The axons show pronounced spreading and filopodial retention on the femur/tibia ( $\mathrm{Fe} / \mathrm{Ti}$ ) boundary (arrowhead). Similar spreading of the axons and a small dorsal branch are seen at the trochanter/femur $(\mathrm{Tr} / \mathrm{Fe}$ ) boundary (solid arrow). The axons typically appear to bend or kink at this point in the pathway. The axons have crossed to the ventral side of the limb along the coxa/trochanter $(\mathrm{Cx} / \mathrm{Tr})$ boundary (open arrow). B, Til pathway in a limb at the same stage of development after treatment with $0.5 \%$ elastase for $2 \mathrm{hr}$. The axons remain spread at the Fe/Ti boundary, although the filopodia appear beaded at the tips, suggesting partial retraction (arrowhead). Several partially retracted filopodia and the characteristic kink in the axons are also retained at the $\mathrm{Tr} / \mathrm{Fe}$ boundary (arrow). Epithelial invaginations mark the $\mathrm{Cx} / \mathrm{Tr}$ (small arrowhead), $\mathrm{Tr} / \mathrm{Fe}$ (double arrowhead), and $\mathrm{Fe} / \mathrm{Ti}$ (broad arrowhead) segment boundaries. $C$, Scanning electron micrograph of axons spread at the Fe/Ti boundary in a limb at the 36-37\% stage of development from which the basal lamina has not been removed. The paired Til axons can be seen between the arrows. The asterisk marks the broadened axonal lamella in the region of the segment boundary (large arrowheads). D, Retention of the Til axons at the $\mathrm{Tr} / \mathrm{Fe}$ boundary in a limb at the $36-37 \%$ stage after partial removal of the basal lamina by treatment with $0.04 \%$ ficin for $70 \mathrm{~min}$. The paired Til axons can be seen between the arrows. The position of the $\mathrm{Tr} / \mathrm{Fe}$ boundary is indicated by the large arrow. Several neuronal processes (arrowheads) extend over the epithelial endfeet and the axons exhibit a broad lamella (asterisk). The Til axons also appear bent in this region. $E$, The Fe/Ti segment boundary after treatment with 
appear to be preserved after treatment with elastase (Fig. 4, $B$, D) or ficin.

A third segment boundary, the coxa/trochanter $(\mathrm{Cx} / \mathrm{Tr})$, is known to have a pronounced effect on the migration of the Ti1 growth cones between 33 and $34 \%$ of development. At this stage, the Til growth cones have reoriented along the $\mathrm{C} x / \operatorname{Tr}$ boundary but are not yet in contact with the Cxl neurons (Keshishian and Bentley, 1983; Caudy and Bentley, 1986b, 1987). Occasionally, limbs are observed in which the presence of the $\operatorname{Tr} 1$ neuron is not indicated by either HRP immunoreactivity or by a response of the Til growth cones to a cell at this position, yet the Til growth cones have reoriented along the $\mathrm{Cx} / \mathrm{Tr}$ boundary. In these limbs, the Ti1 growth cones appear to be interacting primarily with the $\mathrm{Cx} / \mathrm{Tr}$ boundary. We observed limbs of this type in which the association of the Til growth cones with the $\mathrm{Cx} / \mathrm{Tr}$ boundary was retained and the Til somata had assumed a more proximal position after enzymatic treatments known to completely remove the basal lamina (Fig. 4F). This suggests that the interaction between the growth cones and the epithelial cells that constitute the $\mathrm{Cx} / \mathrm{Tr}$ boundary persists after the removal of the basal lamina and that this interaction is sufficiently adhesive to support the proximal relocation of the Til somata.

The behavior of the Til somata in $36-37 \%$ stage limbs after enzyme treatment was also consistent with the suggestion that neurons and segment boundary cells maintain adhesive interactions in the absence of basal lamina. In contrast to the results at the 34-35\% stage, after treatment with elastase or ficin the $\mathrm{Ti} 1$ cell bodies did not reposition proximal to the Fe/ Ti boundary. This was true even if the axons were severely stretched or ruptured by the enzyme-induced elongation of the limb. The stress imposed on the Til axons at this stage by expansion of the limb can be quite large (see Table 2; Materials and Methods). Occasional limbs were observed that were $50 \%$ longer than the average control value.

\section{Interactions of the Til cells with segment boundaries in SEM}

To observe the neural-segment boundary interactions at higher resolution, we examined segment boundary regions in embryos at the $36 \pm 0.5 \%$ stage with scanning electron microscopy. In undigested preparations we observed axons in segment boundary regions that strongly resembled those seen at the light level with the anti-HRP antibody (Fig. 4C). We were unable to preserve the Til axons for SEM inspection after removal of the mesoderm and treatment with standard enzyme concentrations. Embryos from a single clutch of eggs were given identical enzyme treatments, after which some were prepared for SEM and the remainder were fixed and labeled with anti-HRP antibodies. In every animal we observed in SEM after treatment with standard concentrations of elastase or ficin, the basal lamina was completely removed from all positions in the limb, including the segment boundary regions (Fig. $4 E$, Table 1). The animals labeled with anti-HRP antibodies and viewed in whole-mount confirmed that the Til axons were retained at the Fe/Ti segment boundary under conditions known to remove the basal lamina.
In order to preserve the Til axons for SEM inspection, we treated embryos with either elastase $(0.1 \%)$ or ficin $(0.04 \%)$ for $1 \mathrm{hr}$ so as to only partially digest the basal lamina. We observed 3 types of effect on the basal lamina under these conditions: (1) complete removal from all positions in the limb (the Til neurons were not preserved); (2) partial digestion, with no obvious discontinuities at any position along the limb axis; and (3) partial and uneven digestion, with patches of more intact lamina remaining at varying positions. For the majority of cases in which areas of undigested basal lamina remained, these patches were irregularly shaped and their borders were not aligned in any obvious fashion with limb segment boundaries. In a small number of cases, we observed patches with a border that appeared to be aligned on or near a segment boundary. These observations suggest that the behavior of the Til axons at segment boundaries is not dependent on a discontinuity in the basal lamina that is specifically restricted to the segment boundary region.

In some incompletely digested preparations, we observed the Til axons beneath a partially removed basal lamina. In one case, the basal lamina was substantially digested from the region of the $\mathrm{Tr} / \mathrm{Fe}$ segment boundary. The Til axons in this region had a distinct lamellum and numerous filopodia, as well as a kink in the axon, similar to preparations viewed with immunofluorescence (cf. Fig. $4 B$ and $4 D$ ). Similar neuronal-segment boundary interactions were observed at the $\mathrm{Fe} / \mathrm{Ti}$ boundary after partial removal of the basal lamina in $34.5 \%$ embryos (Fig. $3 C$ ). These data strongly suggest that the apposition of the Til axons to the $\mathrm{Fe} / \mathrm{Ti}$, $\mathrm{Tr} / \mathrm{Fe}$, and $\mathrm{Cx} / \mathrm{Tr}$ segment boundaries observed after treatment with elastase and ficin is not due to incomplete removal of the basal lamina in these regions, but rather to an elastase/ficin resistant interaction between the Til neurons and the epithelial cells that constitute the segment boundary.

\section{Discussion}

We have previously shown that mild enzymatic digestion with clastase or ficin removes the basal lamina from grasshopper limb buds, while trypsin leaves the lamina intact (Condic and Bentley, $1988,1989)$. In the work reported here we have investigated the effect of removing the basal lamina when the Til growth cones are in contact with different substrates in the limb, and we have determined the basal lamina dependence of various neural-substrate interactions. We have shown that the Til neurons establish adhesive cell-cell interactions with both neurons and epithelial segment boundary cells that are resistant to elastase/ficin digestion and independent of the basal lamina.

\section{Epithelial elongation}

Elongation of the limb epithelium was observed after incubation with elastase or ficin. This elongation began approximately $1 \mathrm{hr}$ after exposure to the standard concentration of each enzyme and continued for another $1.5 \mathrm{hr}$. Access of the enzymes to the basal surfaces of the epithelium appears to be required for elongation; bath application without opening the dorsal closure was insufficient to induce extension. Elongated epithelia appeared

$0.04 \%$ ficin for $50 \mathrm{~min}$. The basal lamina has been entirely removed from the segment boundary (large arrowheads). Numerous epithelial endfeet remain extended (broad arrowhead). $F$, Til pathway in a limb at the $33.5 \%$ stage of development after treatment with $0.02 \%$ ficin for 2.5 hr. The Til growth cones (open arrow) have reoriented along the $\mathrm{Cx} / \mathrm{Tr}$ segment boundary. Although the growth cones are apparently not in contact with a neuronal soma, they remain extended along the segment boundary and the Til somata have relocated proximally past the Fel neuron $(F)$. The asterisk marks the approximate initial position of the Til somata. Dorsal is $u p$; distal is to the right. Scale bars: $A, B$, and $F, 50 \mu \mathrm{m} ; C, D$, and $E$, $2.5 \mu \mathrm{m}$. 


\section{A: $32 \%$ STAGE}

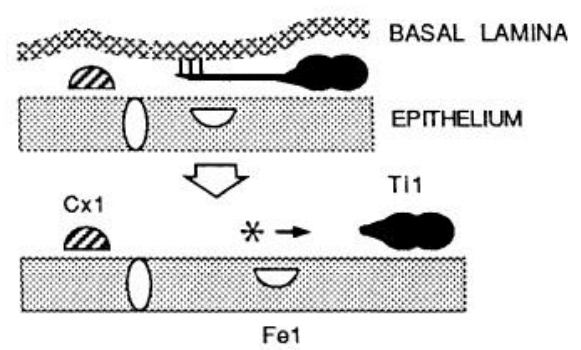

\section{B: $34 \%$ STAGE}

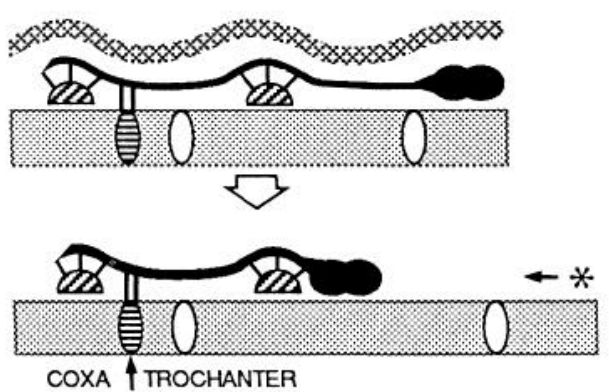

\section{C: $36 \%$ STAGE}
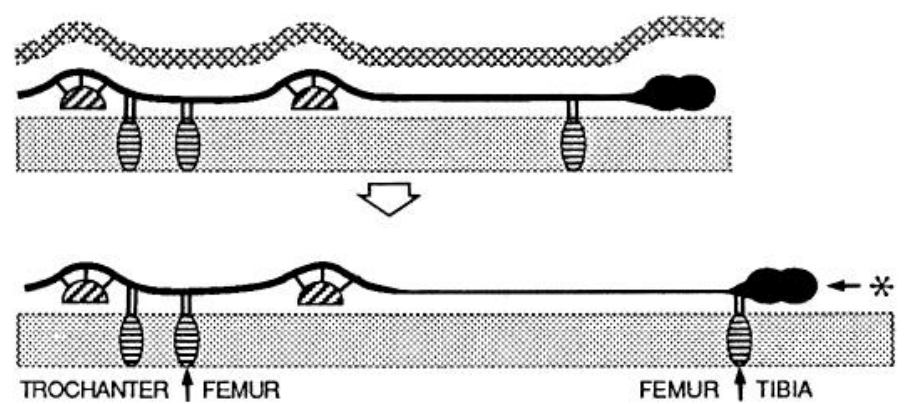

Figure 5. Diagram illustrating the effects of removing the basal lamina at different stages of development. A portion of the limb is viewed perpendicular to its long axis. $\mathrm{Cx} 1, \mathrm{Fel}$, and $\mathrm{Til}$ neurons are included; the basal lamina is indicated by crosshatching; the epithelium is indicated by the stippled box; specialized epithelial cells at the coxa/trochanter $(\mathrm{Cx} / \mathrm{Tr})$, trochanter/femur $(\mathrm{Tr} / \mathrm{Fe})$, and femur/tibia $(\mathrm{Fe} / \mathrm{Ti})$ boundaries are indicated; open segment boundary cell $=$ undifferentiated; horizontal hatched segment boundary cell = differentiated; open neuronal cell = undifferentiated; diagonal hatched neuronal cell $=$ differentiated; the neuronal-substrate attachments that are postulated to underlie the behavior of the Til neurons after the removal of the basal lamina at each stage are indicated by fine lines. A, At the $32 \%$ stage of development, the Til pioneer growth cones have migrated across the intrasegmental epithelium. The Fel neuron has not differentiated (upper panel). When the basal lamina is removed (lower panel), the limb expands slightly and the axons retract to the somata. The asterisk and arrow indicate the initial position of the growth cones and the direction of their retraction. These observations indicate that the Til axons are under tension in vivo. Our results suggest that under the conditions of normal outgrowth, the growth cones are dependent on adhesive interactions with the basal lamina to remain extended over the intrasegmental region of the limb. $B$. At the $34 \%$ stage of development, the Til axons are in contact with both the $\mathrm{Fel}$ and $\mathrm{Cxl}$ neurons and have wrapped them with numerous filopodia (upper panel; see also Fig. 1A). The axons traverse one differentiated $(\mathrm{CX} / \mathrm{Tr})$ and two undifferentiated intact and healthy when inspected in light and scanning electron microscopy.

Enzyme-accelerated elongation of insect epithelia has been reported in imaginal disks of Drosophila (Poodry and Schneiderman, 1971; Fristrom, 1976; Levinson and Bradley, 1984) and Manduca (Nardi et al., 1987). The elongation we observe in grasshopper limb buds after enzymatic treatment appears to differ in at least 2 ways from that reported in other insects. First, the elongation we observe is not an acceleration of a hormonally triggered process that would normally occur over several hours, as is the case for enzyme-accelerated imaginal disk evagination in Manduca and Drosophila. Grasshopper limb buds do elongate during development and eventually reach the lengths we have observed after enzymatic treatment, but this is a gradual process involving considerable cell division over several days time (Bentley et al., 1979). Second, the elongation seen in grasshopper limb buds is correlated with enzymatic treatments known to remove the basal lamina, and is not seen with all proteases. Even at concentrations of trypsin considerably higher than those used for the experiments reported here, consistent limb elongation was not observed. This specificity could reflect a direct relationship between the structural integrity of the lamina and the morphology of the limb, such that disruption of the basal lamina either induces or permits limb elongation. Alternatively, limb elongation could result from enzymatic degradation of specific proteins that are required to maintain epithelial morphology and are more sensitive to digestion by elastase and ficin than to tryptic degradation. In Drosophila imaginal disks, elongation is accomplished equally well with trypsin or chymotrypsin (D. Fristrom, personal communication). Though these observations are suggestive, their import for the role of basal lamina in epithelial morphogenesis during development is unclear (see Fristrom, 1988, for review).

\section{Neural-neural adhesion}

The Til growth cones selectively recognize and reorient towards the cell bodies of other immature neurons as they grow towards the CNS (Bentley and Keshishian, 1982; Ho and Goodman,

\section{$\leftarrow$}

segment boundaries $(\mathrm{Tr} / \mathrm{Fe}$ and $\mathrm{Fe} / \mathrm{Ti})$. After removal of the basal lamina (lower panel), the limb expands slightly and the axons shorten. The Til growth cones remain in contact with the Cxl neurons, and the Til somata relocate proximally. The asterisk and arrow indicate the initial position of the Til somata and the direction of their relocation. The somata relocation indicates that the axons are under tension in vivo and that removing the basal lamina decreases the adhesion of the neuronal somata to the substrate. In addition, the persistence of neural-neural interactions after removal of the basal lamina indicates that these interactions are at least partly adhesive and are mediated by different mechanisms than those responsible for the adherence of the growth cones in the intrasegmental region. $C$. At the $36-37 \%$ stage, the Til axons traverse 3 differentiated segment boundaries $(\mathrm{Cx} / \mathrm{Tr}, \mathrm{Tr} / \mathrm{Fe}$, and $\mathrm{Fe} / \mathrm{Ti}$ ) and frequently extend filopodia and lamellae along these boundaries (upper panel; see also Fig. 4A). After removal of the basal lamina (lower panel), the limb expands significantly and the axons are stretched. Interactions of the Til cells with the other neurons in the limb and with the limb segment boundaries are retained after removal of the lamina. The Til somata relocate to the Fe/Ti segment boundary, but do not move beyond this boundary despite severe stretching of the axons due to limb elongation. The asterisk and arrow indicate the initial position of the Til somata and the direction of their relocation. These observations suggest that the interations between the Til neurons and mature segment boundaries are at least partly adhesive and are independent of the basal lamina. 
1982; Keshishian and Bentley, 1983). The initial filopodial contact between the Til neurons and neuronal guidepost cells may result in an active redirection of the growth cones (Caudy and Bentley, 1986b). Growth cones are dependent on the presence of filopodia for normal pathfinding (Bentley and Toroian-Raymond, 1986). Once initial contact has been established, the Til growth cones will often wrap the cell bodies of the guidepost neurons with numerous filopodia and occasionally spread lamellae over them as well (Caudy and Bentley, 1986b).

The nature of growth cone affinity for other neurons in the limb has not been previously investigated. If the basal lamina was removed after the Til cells were in contact with the Cxl neuronal somata, the growth cones remained tightly apposed to the $\mathrm{Cx} 1$ cells. The interactions between the Til growth cones and the $\mathrm{Cx} 1$ cell bodies are sufficiently adhesive both to anchor the growth cones (thereby preventing growth cone retraction) and to permit the translocation of the Til somata over considerable distances. It has been demonstrated in vitro that when growth cones are strongly attached to a substrate, they are able to "tow" their more weakly adhering somata behind them as they migrate (Bray, 1987). These data indicate that the Til-Cx 1 neuronal interactions observed in vivo at this stage are at least partly mediated by strongly adhesive, cell-cell contacts that are resistant to elastolytic degradation and independent of the basal lamina.

It is well established that neurons in culture express a number of specific cell-cell recognition molecules, many of which are thought to mediate adhesive interactions between neurons in vitro (reviewed, Obrink, 1986; Jessell, 1988). Moreover, growth cones can employ extracellular matrix receptors as well as specific cell-cell adhesion molecules for migration on cell surfaces (Bixby et al., 1987; Tomaselli et al., 1987). Cell adhesion molecules in insects have not been extensively characterized. However, several cell-surface glycoproteins found on insect neurons are currently under investigation (Bastiani et al., 1987; Snow et al., 1987). Although the molecular basis of neuronal-neuronal interaction in the grasshopper limb remains obscure, the fact that contacts between the Til growth cones and the $\mathrm{Cx} 1$ somata are maintained despite the force exerted on them by the translocation of the Til somata constitutes proof of the adhesive nature of these connections. This in no way implies that the neuronalneuronal interactions underlying growth cone reorientation during axon outgrowth are strictly or exclusively adhesive. On a cellular and molecular level, the interactions between neurons in this system could prove to be quite complex. However, neuronal-neuronal interaction in this system clearly involves the establishment of strong, adhesive contacts.

\section{Neural-segment boundary adhesion}

The growth cones of the Til neurons and other neurons of the limb respond selectively to limb segment boundaries. These responses constitute an important element of axon guidance in this system (Bentley and Caudy, 1983b; Caudy and Bentley, 1986a, 1987). Neuronal interactions with epithelial cells and/ or epithelial basal lamina are also crucial to axon guidance in Drosophila wing disks (Blair and Palka, 1985a, b, 1987; Palka, 1987). The response of the Til axons to the femur/tibia (Fe/ $\mathrm{Ti})$ and the trochanter/femur $(\mathrm{Tr} / \mathrm{Fe})$ segment boundaries changes during development (Caudy and Bentley, 1986a, 1987). The changes in axonal spreading and filopodial retention observed at segment boundaries suggest that the relative affinity of bound- aries for axonal membrane increases over time. Whether neuronal affinity is due to a differential interaction of the neurons with the boundary cells themselves or due to qualitative and/ or quantitative discontinuities in the basal lamina associated with these regions was not clear from our previous observations. We have now determined that the Ti1 axons remain spread at the $\mathrm{Fe} / \mathrm{Ti}$ and $\mathrm{Tr} / \mathrm{Fe}$ segment boundaries under enzymatic conditions known to remove the basal lamina. The anchoring of the Til axons at the Fe/Ti segment boundary observed by $37 \%$ of development is sufficiently adhesive to prevent the translocation of the Til somata proximal to this point. This is true despite the severe stretching of the axons (occasionally to the point of rupture) that results from the enzyme-induced expansion of the limb epithelium at this stage (Table 2). In addition, in a small number of younger embryos, the Til somata relocate proximally after removal of the basal lamina when the growth cones appeared to be in contact only with the $\mathrm{Cx} / \mathrm{Tr}$ segment boundary (see Fig. $4 F$ ). These observations strongly suggest that neuronal affinity for limb segment boundaries is due at least in part to a direct, adhesive interaction between neurons and the specialized epithelial cells that constitute the boundary.

There are several lines of evidence to suggest that epithelial cells constituting segment boundaries in insects have unique anatomical (Lawrence, 1975), physiological (Blennerhasset and Caveney, 1984), behavioral (Campbell and Shelton, 1987), and cell-surface (Caudy and Bentley, 1986c, 1987) properties. After enzymatic removal of the basal lamina, the interactions of the Til neurons with segment boundary epithelia persist, while those with intrasegmental epithelia do not (Condic and Bentley, 1989). The Ti1 growth cones selectively recognize and reorient along segment boundaries as they migrate in vivo (Caudy and Bentley, 1986a, 1987). The growth cone-segment boundary adhesion we have demonstrated here may constitute an important element of this response.

\section{Til adhesive interactions after removal of the basal lamina}

Removing the basal lamina from grasshopper limbs alters the adhesion of the Til growth cones and axons to some substrata in the limb while leaving other ncural-substratc interactions unperturbed. The effects of removing the basal lamina when the Ti1 neurons are in contact with different cellular and acellular substrates in the limb are summarized in Figure 5. Our results suggest that initial axonal outgrowth occurs through growth conebasal lamina interactions that are sufficiently adhesive to resist the tension generated in the Til axons by cytoskeletal actin filaments. Removing the basal lamina at this stage results in the retraction of the axons to the Til somata (Fig. $5 A$; Condic and Bentley, 1989). Later in development, the Til neurons establish cell-cell adhesive interactions with both neurons and segment boundary cells that are independent of the basal lamina (Fig. 5, $B, C)$. These adhesive contacts may constitute an important element of axon guidance in this system.

\section{References}

Ashhurst, D. E. (1965) The connective tissue sheath of the locust nervous system: Its development in the embryo. Q. J. Microsc. Soc. 106: 61-73.

Ashhurst, D. E. (1982) The structure and development of insect connective tissues. In Insect Ultrastructure, R. C. King and H. Akai, eds., pp. 313-350, Plenum, New York.

Ball, E. E., and C. S. Goodman (1985) Muscle development in the 
grasshopper embryo. II. Syncytial origin of the extensor tibiae muscle pioneers. Dev. Biol. 111: 399-416.

Ball, E. E., H. G. deCouet, P. L. Horn, and J. M. A. Quinn (1987) Hacmocytcs sccrete basement membrane components in embryonic locusts. Development 99: 255-259.

Bastiani, M. J., A. L. Harrelson, P. M. Snow, and C. S. Goodman (1987) Expression of fasciclin I and II glycoproteins on subsets of axon pathways during neuronal development in the grasshopper. Cell 48: 745755.

Bate, C. M. (1976) Pioneer neurons in an insect embryo. Nature 260: 54-56.

Bentley, D., and M. Caudy (1983a) Pioneer axons lose directed growth after selective killing of guidepost cells. Nature $260: 62-65$.

Bentley, D., and M. Caudy (1983b) Navigational substrates for peripheral pioneer growth cones: Limb-axis polarity cues, limb-segment boundaries, and guidepost neurons. Cold Spring Harbor Symp. Quant. Biol. 48: 573-585.

Bentlcy, D., and H. Kcshishian (1982) Pathfinding by peripheral pioneer neurons in grasshoppers. Science 218: 1082-1088.

Bentley, D., and A. Toroian-Raymond (1986) Disoriented pathfinding by pioneer neurone growth cones deprived of filopodia by cytochalasin treatment. Nature 323: 712-715.

Bentley, D., H. Keshishian, M. Shankland, and A. Toroian-Raymond (1979) Quantitative staging of embryonic development of the grasshopper, Schistocerca nitens. J. Embryol. Exp. Morphol. 54: 47-74.

Bixby, J. L., R. S. Pratt, J. Lilien, and L. F. Reichardt (1987) Neurite outgrowth on muscle cell surfaces involves extracellular matrix receptors as well as $\mathrm{Ca}^{2+}$-dependent and -independent cell adhesion molecules. Proc. Natl. Acad. Sci. USA 84: 2555-2559.

Blair, S. S., and J. Palka (1985) Axon guidance in cultured wing discs and disc fragments of Drosophila. Dev. Biol. 108: 411-420.

Blair, S. S., M. A. Murray, and J. Palka (1985) Axon guidance in cultured epithelial fragments of Drosophila wing. Nature 315: 406408.

Blair, S. S., M. Murray, and J. Palka (1987) The guidance of axons from transplanted neurons through aneural Drosophila wings. J. Neurosci. 7: 4165-4175.

Blennerhassett, M. G., and S. Caveney (1984) Separation of developmental compartments by a cell type with reduced junctional permeability. Nature 309: 361-364.

Blumberg, B., A. J. MacKrell, P. F. Olson, M. Kurkinen, J. M. Monson, J. E. Natzle, and J. H. Fessler (1987) Basement membrane procollagen IV and its specialized carboxyl domain are conserved in Drosophila, mouse and human. J. Biol. Chem. 262: 5947-5950.

Bogaert, T., N. Brown, and M. Wilcox (1987) The Drosophila ps2 antigen is an invertebrate integrin that, like the fibroncetin receptor, becomes localized to muscle attachments. Cell 51: 929-940.

Bray, D. (1987) Growth cones: Do they pull or are they pushed? Trends Neurosci. 10: 431-434.

Campbell, G. L., and P. M. J. Shelton (1987) Cell behavior during postembryonic pattern regulation in the insect abdomen (Oncopeltus fasiatus). I. Regeneration of segment borders. Development 101:221235.

Caudy, M., and D. Bentley (1986a) Pioneer growth cone morphologies reveal proximal increases in substrate affinity within leg segments of grasshopper embryos. J. Neurosci. 6: 364-379.

Caudy, M., and D. Bentley (1986b) Pioneer growth cone steering along a series of neuronal and non-neuronal cues of different affinities. J. Neurosci. 6: 1781-1795.

Caudy, M., and D. Bentley (1986c) Epithclial cell spccialization at a limb segment boundary in the grasshopper embryo. Dev. Biol. 118: 399-402.

Caudy, M., and D. Bentley (1987) Pioneer growth cone behavior at a differentiating limb segment boundary in the grasshopper embryo. Dev. Biol. 119: 454-465.

Condic, M. L., and D. Bentley (1988) Effects of enzymatic removal of the basal lamina on pioneer neurons in grasshopper embryos. Soc. Neurosci. Abstr. 14: 451.

Condic, M. L., and D. Bentley (1989) Removal of the basal lamina in vivo reveals growth cone-basal lamina adhesive interactions and axonal tension in grasshopper embryos. J. Neurosci. 9: 2678-2686.

Fessler, L. I., A. G. Campbell, K. G. Duncan, and J. H. Fessler (1987) Drosophila laminin: Characterization and localization. J. Cell Biol. 105: 2383-2391.

Fristrom, D. (1976) The mechanism of evagination of imaginal discs of Drosophila melanogaster. III. Evidence for cell rearrangement. Dev. Biol. 54: 163-171.

Fristrom, D. (1988) The cellular basis of epithelial morphogenesis Tissue Cell 20 (in press).

Grateios, D., C. Naidet, M. Astier, J. P. Thiery, and M. Semeriva (1988) Drosophila fibronectin: A protein that shares properties similar to those of its mammalian homologue. EMBO J. 7: 215-223.

Hay, E. D. (1981) Collagen and embryonic development. In Cell Biology of Extracellular Matrix, E. D. Hay, ed., pp. 379-409, Plenum, New York

Ho, R. K., and C. S. Goodman (1982) Peripheral pathways are pioneered by an array of central and peripheral neurons in grasshopper embryos. Nature 297: 404-406.

Hynes, R. O. (1987) Integrins: A family of cell surface receptors. Cell 48: 549-554.

Jan, L. Y., and Y. N. Jan (1982) Antibodies to horseradish peroxidase as specific neuronal markers in Drosophila and grasshopper embryos. Proc. Natl. Acad. Sci. USA 79: 2700-2704.

Jessell, T. M. (1988) Adhesion molecules and the hierarchy of neural development. Neuron $1: 3-13$.

Keshishian, H. (1980) The origin and morphogenesis of pioneer neurons in the grasshopper metathoracic leg. Dev. Biol. 80: 388-397.

Keshishian, H., and D. Bentley (1983) Embryogenesis of peripheral nerve pathways in grasshopper legs. Dev. Biol. 96: 98-124.

Lawrence, P.A. (1975) The structure and properties of a compartment border: The intersegmental boundary in Oncopeltus. In Ciha Foundation Symposium 29: Cell Patterning, pp. 3-23, Elsevier, New York.

Lefcort, F., and D. Bentley (1987) Pathfinding by pioneer neurons in isolated, opened and mesoderm-free limb buds of embryonic grasshoppers. Dev. Biol. 119: 466-480.

Lefcort, F., and D. Bentley (1989) Organization of cytoskeletal elements and organelles preceding growth cone initiation in an identified neuron in situ. J. Cell Biol. 108: 1737-1749.

Leptin, M., R. Aebersold, and M. Wilcox (1987) Drosophila positionspecific antigens resemble the vertebrate fibronectin-receptor family. EMBO J. 6: 1037-1043.

Levinson, G., and T. J. Bradley (1984) Removal of insect basal laminae using elastase. Tissue Cell 16: 367-375

MacKrell, A. J., B. Blumberg, S. R. Haynes, and J. H. Fessler (1988) The lethal myospheroid gene of Drosophila encodes a membrane protein homologous to vertebrate integrin B subunits. Proc. Natl. Acad. Sci. USA 85: 2633-2637.

Mirre, C., J.-P. Cecchini, Y. Le Parco, and B. Knibiehler (1988) Do novo expression of a type IV collagen gene in Drosophila embryos is restricted to mesodermal derivatives and occurs at germ band shortening. Development 102: 369-376.

Montell, D. J., and C. S. Goodman (1988) Drosophila substrate adhesion molecule: Sequence of laminin B1 chain reveals domains of homology with mouse. Cell 53: 463-473.

Nardi, J. B., S.-W. Norby, and S. M. Magee-Adams (1987) Cellular events within peripodial epithelia that accompany evagination of Manduca wing discs: Conversion of cuboidal epithelia to columnar epithelia. Dev. Biol. 119: 20-26.

Obrink, B. (1986) Epithelial cell adhesion molecules. Exp. Cell Res. 163: 1-21.

Palka, J. (1987) Axon guidance in the insect periphery. Development 99: $307-310$

Poodry, C. A., and H. A. Schneiderman (1971) Intercellular adhesivity and pupal morphogenesis in Drosophila melanogaster. Wilhelm Roux's Arch. Dev. Biol. 168: 1-9.

Sanes, J. R., and A. Y. Chiu (1983) The basal lamina of the neuromuscular junction. Cold Spring Harbor Symp. Quant. Biol. 48: 667678.

Sanes, J. R., and J. Covault (1985) Axon guidance during reinnervation of skeletal muscle. Trends Neurosci. 8: 525-528.

Snow, P. M., N. H. Patel, A. L. Harrelson, and C. S. Goodman (1987) Neural-specific carbohydrate moiety shared by many surface glycoproteins in Drosophila and grasshopper embryos. J. Neurosci. 7: $4137-$ 4144.

Tomaselli, K. J., C. H. Damsky, and L. F. Reichardt (1987) Interactions of a neuronal cell line ( $\mathrm{pc12}$ ) with laminin, collagen IV and fibronectin: Identification of integrin-related glycoproteins involved in attachment and process outgrowth. J. Cell Biol. 105: 2347-2358.

Wigglesworth, V. B. (1953) The origin of sensory neurones in an insect Rhodnius prolixus (Hemiptera). Q. J. Microsc. Sci. 94: 93-112. 